Les parents de jeunes de 15 ans connaissent-ils les camarades d'école de leur enfant et leurs parents ?

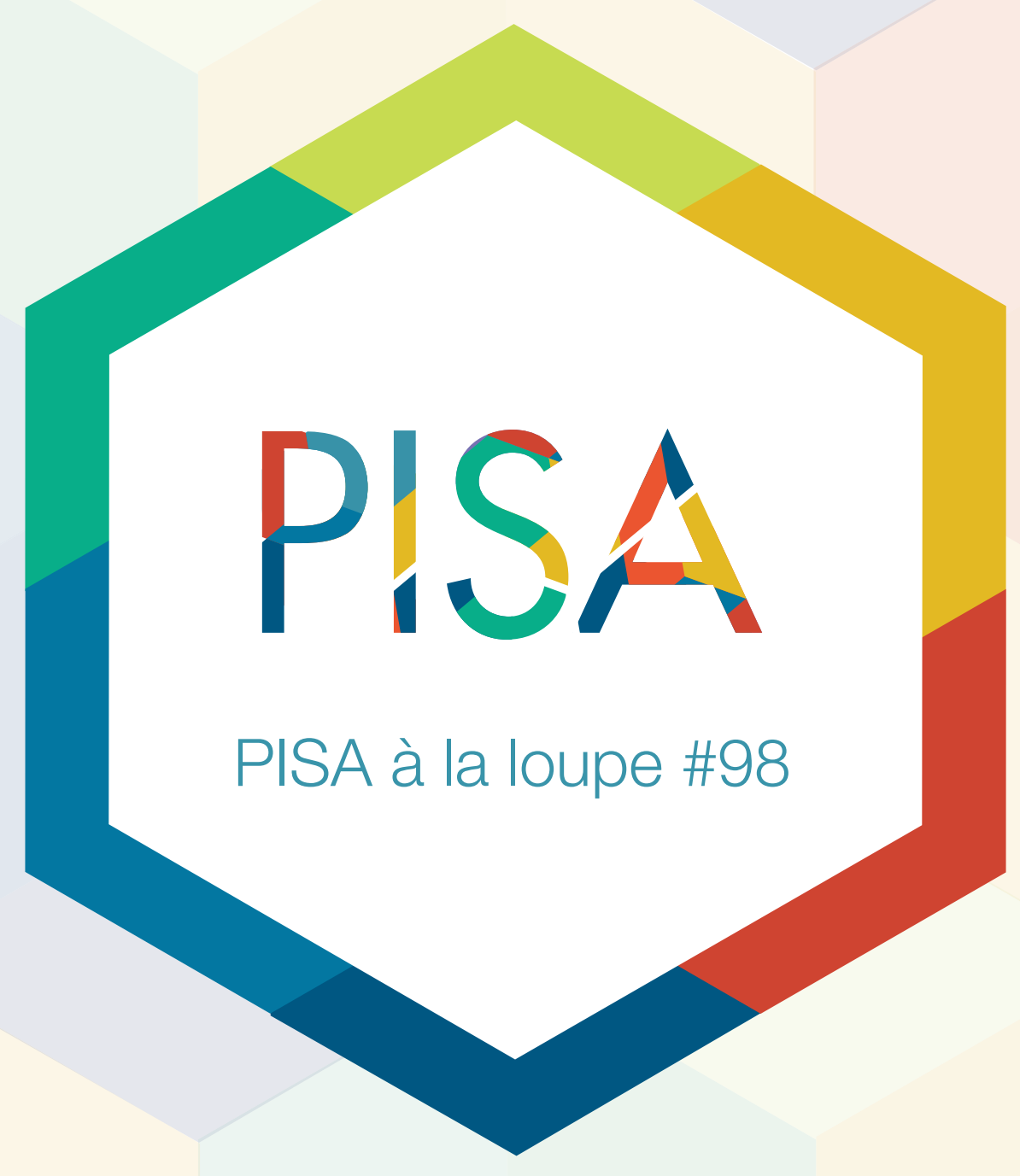




\section{Les parents de jeunes de 15 ans connaissent-ils les camarades d'école de leur enfant et leurs parents?}

- En moyenne, dans les pays de l'OCDE, les parents d'élèves de 15 ans indiquent qu'ils connaissent le nom de cinq camarades de leur enfant et quatre parents de ces camarades. Les parents en Espagne, en Géorgie et en Irlande sont ceux qui connaissent le plus de camarades et de parents, tandis que les parents en France, à Hong Kong (Chine) et à Macao (Chine) sont ceux qui en connaissent le moins.

- Dans la plupart des pays et des économies, les parents d'enfants qui sont scolarisés dans des établissements favorisés sur le plan socio-économique connaissent davantage de camarades de leur enfant et de parents de ces camarades que les parents d'enfants d'établissements défavorisés.

- Les élèves dont les parents connaissent un plus grand nombre de leurs camarades et de parents de ces derniers obtiennent de meilleurs résultats en matière de résolution collaborative de problèmes, et subissent moins de harcèlement, même après avoir pris en compte le statut socio-économique.

Les parents tissent souvent des relations enrichissantes avec les enseignants, les élèves et d'autres parents d'élèves dans les établissements scolaires de leur enfant. Ils peuvent ainsi nouer de nouvelles amitiés et contribuer au parcours scolaire de leur enfant, mais peuvent surtout contribuer indirectement à un environnement favorable dans l'établissement, en édifiant des normes de comportement plus strictes, en communiquant des informations importantes, en favorisant la confiance et en créant des passerelles entre l'établissement et la collectivité dans son ensemble.

Lors de l'enquête PISA, il a été demandé aux parents des dix-huit pays et économies ayant administré le questionnaire " Parents " d'indiquer le nombre de camarades de leur enfant dont ils connaissent le nom et le nombre de parents de ces camarades qu'ils connaissent. En moyenne, dans les systèmes d'éducation de l'OCDE qui ont administré le questionnaire "Parents », les parents indiquent qu'ils connaissent environ cinq camarades de leur enfant et quatre parents de ces camarades. On constate toutefois des différences significatives entre les pays et les économies. Les parents en Espagne, en Géorgie et en Irlande sont ceux qui connaissent le plus de camarades et de parents, tandis que les parents en Corée, à Hong Kong (Chine) et à Macao (Chine) sont ceux qui en connaissent le moins. En Géorgie, par exemple, les parents indiquent qu'ils connaissent en moyenne six autres parents d'élèves, soit trois fois plus que le nombre indiqué par les parents à Hong Kong (Chine).

Dans certains pays, il est plus naturel pour les parents de nouer des liens avec d'autres personnes de la communauté scolaire, mais d'autres facteurs peuvent expliquer les différences qui sont observées entre les pays en ce qui concerne le nombre de connaissances des parents dans les établissements scolaires. En Espagne, par exemple, les élèves de 15 ans dans les établissements publics doivent en général changer une fois d'établissement au cours de leur parcours, vers l'âge de 12 ans, alors qu'en France, ils doivent changer d'établissement au moins trois fois, autour de 6 ans, 11 ans et 15 ans. Les élèves espagnols et leurs parents ont donc plus de possibilités de nouer des relations stables que leurs homologues français, car il faut du temps pour se faire des amis. 


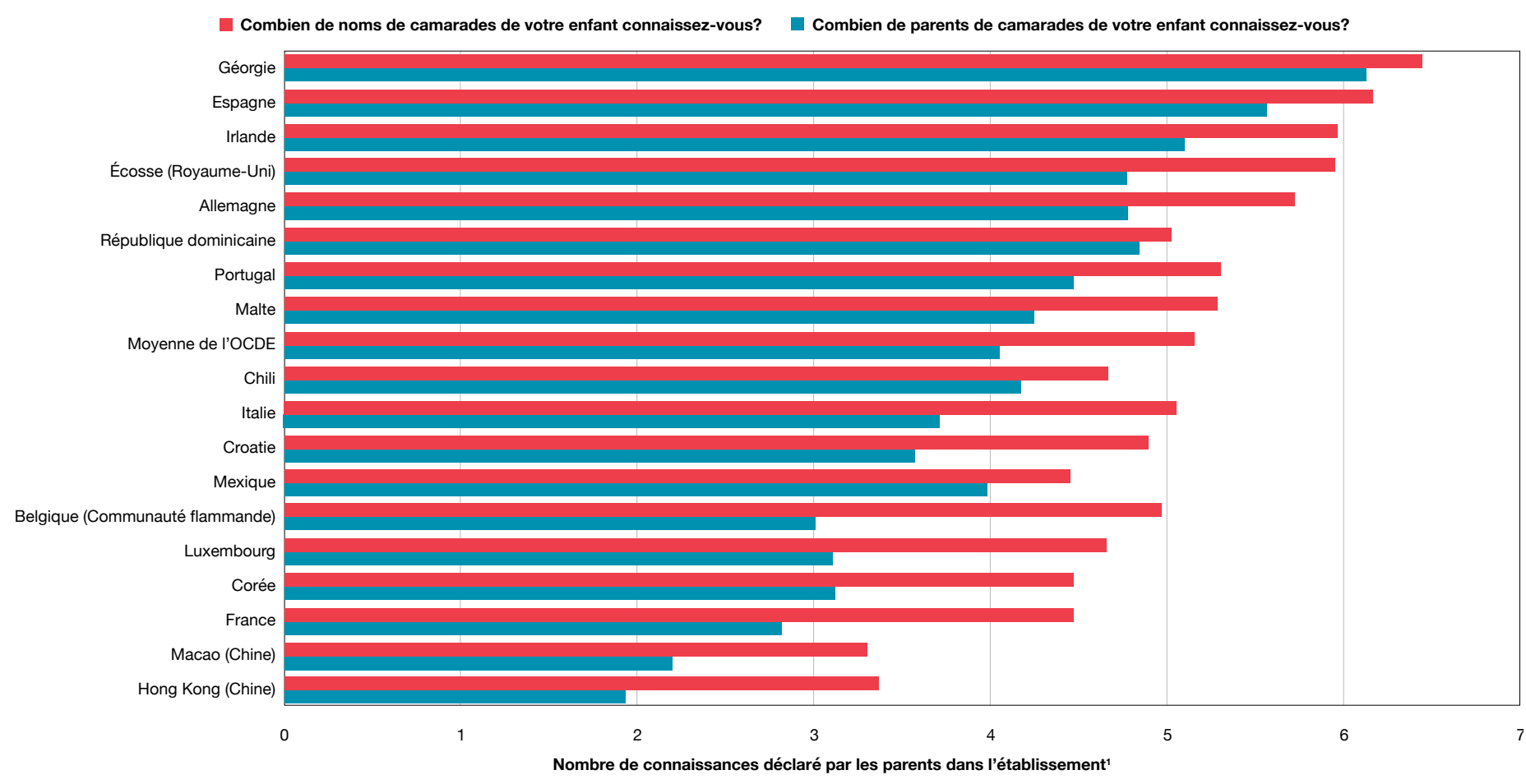

1. Une valeur de " 7 " a été attribuée aux parents qui ont répondu « 6 ou plus ".

Remarque : Seuls les pays et les économies qui ont administré le questionnaire « Parents " figurent sur le graphique.

Les pays et économies sont classés par ordre décroissant du nombre de connaissances.

Source : OCDE, Base de données PISA 2015.

La participation des parents aux activités scolaires, notamment leur participation aux conférences pour les parents, peut aussi influer sur leur connaissance d'autres parents dans l'établissement de leur enfant. Dans les systèmes scolaires dans lesquels les parents des élèves de 15 ans connaissent le plus grand nombre de camarades de leur enfant et de parents de ces camarades, y compris en Allemagne, en Écosse (Royaume-Uni), en Espagne, en Géorgie et en Irlande, environ huit parents sur dix ont participé à une réunion ou à une conférence destinée aux parents qui a été programmée au cours de l'année scolaire écoulée, tandis que dans les pays dans lesquels les parents ont le moins de connaissances, notamment en Corée, en France, à Hong Kong (Chine) et à Macao (Chine), moins de sept parents sur dix ont participé à ces événements. À Hong Kong (Chine), seuls trois parents sur dix ont participé à une réunion ou à une conférence pour les parents qui a été programmée.

Dans la plupart des pays et des économies ayant administré le questionnaire, les parents d'enfants qui sont scolarisés dans des établissements favorisés sur le plan socio-économique connaissent davantage de camarades d'établissement de leur enfant et d'autres parents que les parents d'enfants d'établissements défavorisés. Cet écart socio-économique est particulièrement important dans les systèmes éducatifs dans lesquels les parents indiquent qu'ils ont moins de connaissances, en moyenne, dans l'établissement scolaire, par exemple en Communauté flamande de Belgique, à Hong Kong (Chine), au Luxembourg et à Macao (Chine), mais aussi au Chili et à Malte. II est intéressant de constater qu'au Mexique et dans une moindre mesure en République dominicaine, en Géorgie et au Portugal les parents d'élèves d'établissements favorisés connaissent moins de camarades de leur enfant et d'autres parents que les parents d'enfants scolarisés dans des établissements défavorisés. 


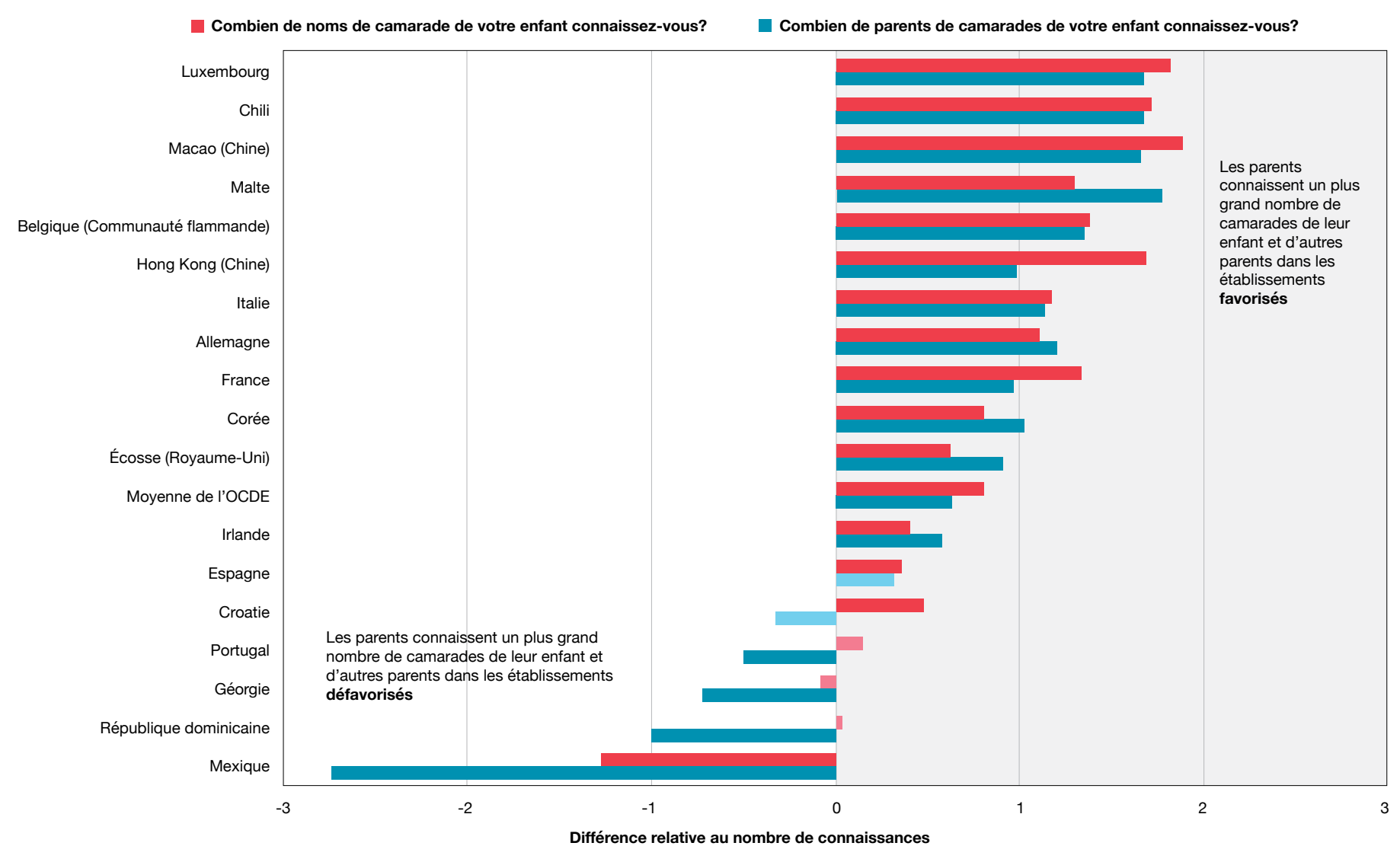

Remarques : Les écarts statistiquement significatifs sont indiqués en couleur plus foncée.

Seuls les pays et les économies qui ont administré le questionnaire "Parents " figurent sur le graphique.

Les pays et économies sont classés par ordre décroissant de différence du nombre de connaissances des parents dans les établissements.

Source : OCDE, Base de données PISA 2015.

\section{Les enfants dont les parents entretiennent des relations sociales peuvent en tirer de nombreux bienfaits}

Les élèves dont les parents connaissent leurs camarades et d'autres parents - une situation généralement désignée par l'expression « connexions intergénérationnelles »- peuvent en tirer des bénéfices. Si l'enquête PISA ne permet pas d'établir de relations de cause à effet, ses résultats sont conformes à cette attente. Le premier rapport PISA sur la résolution collaborative de problèmes montre, par exemple, que dans les pays de l'OCDE, en moyenne, les élèves de 15 ans obtiennent de meilleurs résultats en résolution collaborative de problèmes et valorisent davantage les relations et le travail d'équipe lorsque leurs parents connaissent davantage leurs camarades et les parents de ces derniers, même après avoir pris en compte le statut socio-économique des élèves et des établissements. Par exemple, pour chaque camarade de classe supplémentaire connu par leurs parents, les élèves obtiennent deux points de plus en résolution collaborative de problèmes.

Ce scénario peut être particulièrement utile pour endiguer et empêcher le harcèlement. Un harceleur peut réfléchir à deux fois avant de menacer un autre élève, se moquer de lui ou le blesser s'il sait que leurs parents se connaissent. Les enfants harcelés peuvent également se sentir plus protégés lorsque leurs parents entretiennent des relations avec d'autres parents et avec le personnel de l'établissement scolaire. 
Et, point important s'il en est, la définition et l'application de normes uniformes pour lutter contre le harcèlement devraient être facilitées lorsque les parents se connaissent et connaissent les camarades de leurs enfants. Les résultats de l'enquête PISA montrent qu'en moyenne, dans les pays de l'OCDE ayant administré le questionnaire "Parents ", les élèves dont les parents connaissent un plus grand nombre de parents de leurs camarades sont moins victimes de harcèlement, notamment de formes de harcèlement relationnel, même après avoir pris en compte le statut socioéconomique. Par exemple, la probabilité d'être écarté volontairement au moins quelques fois par mois diminue de six points de pourcentage pour chaque parent de camarade supplémentaire faisant partie des connaissances des parents d'élève. Les élèves sont également moins nombreux à indiquer qu'ils ont été victimes de harcèlement, notamment de harcèlement physique, lorsque leurs parents connaissent un plus grand nombre de leurs camarades par leur nom. À titre d'exemple, les élèves dont les parents indiquent qu'ils ne connaissent aucun des camarades de leur enfant sont $50 \%$ plus susceptibles d'être frappés ou bousculés par d'autres élèves quelques fois par mois que les élèves dont les parents indiquent qu'ils connaissent cinq de leurs camarades.

\section{Connaissance des parents dans l'établissement et harcèlement Moyenne de l'OCDE}

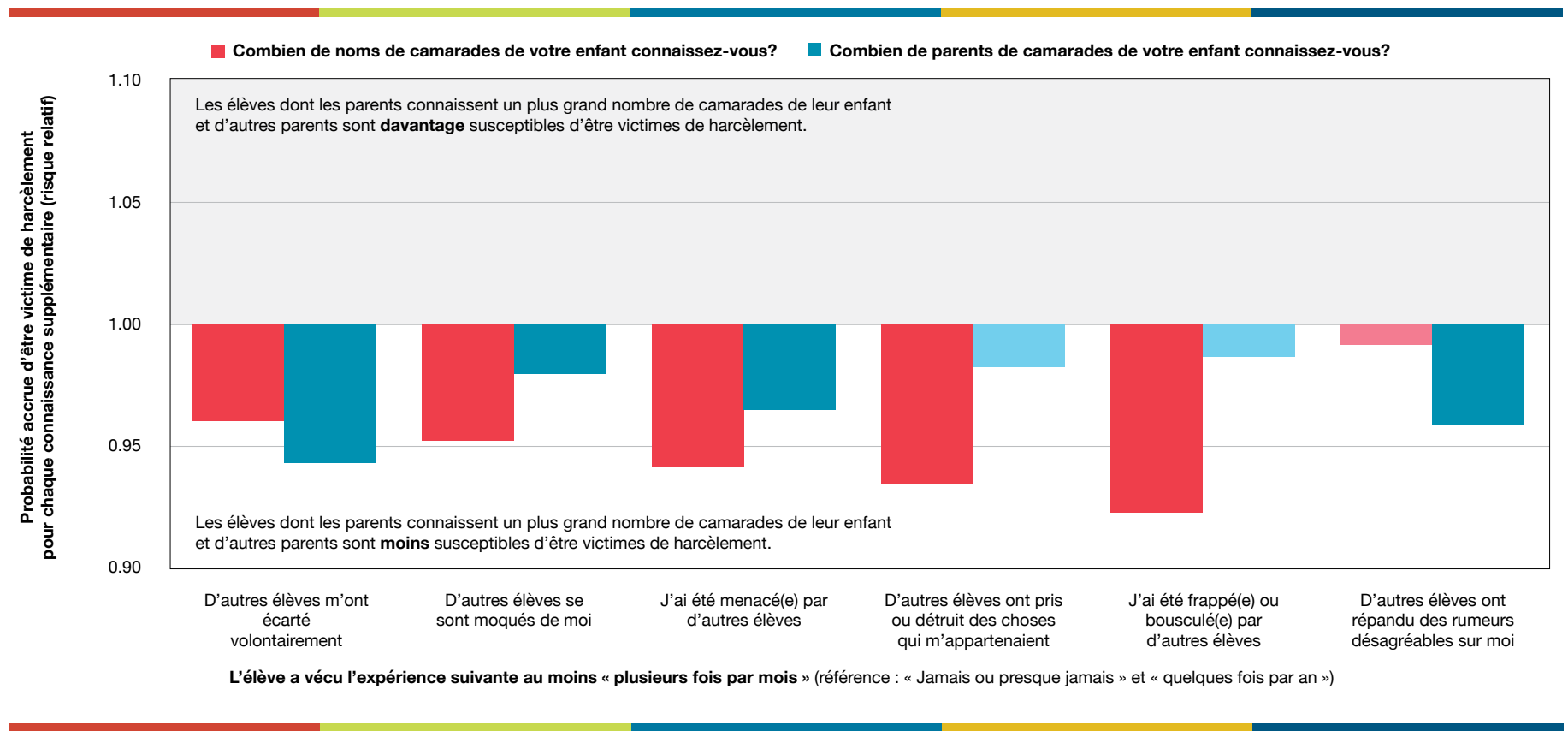

Remarques : Les écarts statistiquement significatifs sont indiqués en couleur plus foncée.

Risque relatif, après avoir pris en compte le statut socio-économique.

La moyenne de l'OCDE inclut seulement les pays qui ont administré le questionnaire " Parents ".

Source : OCDE, Base de données PISA 2015.

\section{Pour conclure}

Dans les établissements dans lesquels les parents connaissent les camarades de leur enfant et leur famille, les élèves sont davantage susceptibles de perfectionner leurs compétences, d'améliorer leurs attitudes en matière de collaboration et de se sentir plus heureux et plus en sécurité à l'école. Ces connaissances évoluent souvent naturellement en interactions quotidiennes et peuvent se transformer en véritables amitiés. Pour encourager ces relations, les établissements peuvent commencer par offrir une atmosphère accueillante. Ils peuvent organiser des fêtes et des conférences pour les familles des élèves, donner aux parents la possibilité de participer aux activités scolaires et établir des normes informelles pour le comportement des élèves. 


\section{Pour tout complément d'information}

\section{Contacter : Alfonso Echazarra (Alfonso.Echazarra@oecd.org)}

Consulter : OCDE (2017), Résultats du PISA 2015 (Volume V) : Résolution collaborative de problèmes, PISA, Éditions OCDE, Paris, https://doi.org/10.1787/9789264305199-fr.

Le mois prochain : La réussite scolaire peut-elle aider les élèves défavorisés à accéder à un niveau d'études supérieur à celui de leurs parents?

Ce document est publié sous la responsabilité du Secrétaire général de l'OCDE. Les opinions et les arguments exprimés ici ne reflètent pas nécessairement les vues officielles des pays membres de l'OCDE.

Ce document, ainsi que les données et cartes qu'il peut comprendre, sont sans préjudice du statut de tout territoire, de la souveraineté s'exerçant sur ce dernier, du tracé des frontières et limites internationales, et du nom de tout territoire, ville ou région.

Ce texte est disponible sous licence Attribution - Pas d'Utilisation Commerciale - Partage dans les Mêmes Conditions 3.0 Organisations Internationales (CC BY-NC-SA 3.0 IGO) Pour toute information spécifique quant à l'étendue et aux termes de la licence ainsi que d'une possible utilisation commerciale de ce texte et pour toute utilisation de données PISA, prière de consulter les Conditions d'utilisation à www.oecd.org/fr/conditionsdutilisation. 